Series A

I. MATHEMATICA

509

\title{
MODULUS AND CAPACITY INEQUALITIES FOR QUASIREGULAR MAPPINGS
}

BY

JUSSI VÄISÄL $\ddot{A}$ 
Academia Scientiarum Fennica

Copyright (C) 1972 by

ISBN $951-41-0036-0$

Communicated 11 February 1972 


\section{Introduction}

One of the most important tools in the theory of quasiconformal mappings is the double inequality

$$
\frac{1}{K} M(\Gamma) \leq M(f \Gamma) \leq K M(\Gamma)
$$

valid for every $K$-quasiconformal mapping $f: G \rightarrow G^{\prime}$ and for every path family $\Gamma$ in $G$. The left hand inequality is not always true for $K$-quasiregular mappings. (For terminology, see [4].) However, by a result of Poleckii [7, Theorem 1], the right hand inequality also holds in this more general case. A related result for condensers was given in [4, 7.1]. Poleckii [7, Theorem 2] also proved that the stronger inequality

$$
M\left(\Gamma^{\prime}\right) \leq \frac{K}{m} M(\Gamma)
$$

is true in the following case: $D$ is a normal domain of $f, m=N(f, D)$ is the multiplicity of $f$ in $D, \Gamma^{\prime}$ is a family of injective paths in $f D$, and $\Gamma$ is the family of all paths $\gamma$ in $D$ such that $f \circ \gamma \in \Gamma^{\prime}$. A related result for condensers was given by Martio [3, 5.1 and 5.13]. Martio and Poleckii applied their inequalities to study the local behavior of a quasiregular mapping.

The main purpose of this paper is to establish (1.2) in a more general situation. In particular, the paths of $\Gamma$ need not lie in a compact part of $G$. We also give the corresponding result for condensers. The inequality is applied to study the behavior of a mapping at an isolated singularity.

Our terminology and notation is the same as in [4]. In particular, the notation $f: G \rightarrow R^{n}$ includes the assumption that $G$ is a domain in $R^{n}$ and that $f$ is continuous.

\section{Preliminary results}

2.1. Lemma. Suppose that $f: G \rightarrow R^{n}$ is discrete and open, that $E \subset G^{\prime}$ is compact, and that $y_{0} \in f E \backslash f\left(B_{f} \cap E\right)$. Then there is a neighborhood $V_{\mathbf{0}}$ 
of $y_{0}$ such that for every connected neighborhood $V \subset V_{0}$ of $y_{0}$, the following conditions are satisfied:

(1) $V \cap f\left(B_{f} \cap E\right)=\varnothing$.

(2) The components of $f^{-1} V$ which meet $E$ form a finite collection $D_{1}, \ldots, D_{k}$.

(3) $f$ defines homeomorphisms $f_{i}: D_{i} \rightarrow \mathrm{I}$.

Proof. This is essentially the same as $[4,7.15]$. Thus let $U_{1}, \ldots, U_{k}$ be disjoint neighborhoods of the points in $E \cap f^{-1}\left(y_{0}\right)$ such that $\bar{\Gamma}_{i} \subset G$ and such that $f \mid \bar{U}_{i}$ is injective. Then

$$
V_{0}=\left(\bigcap_{i=1}^{k} f U_{i}\right) \backslash f\left(E \backslash \bigcup_{i=1}^{k} U_{i}\right)
$$

is the required neighborhood of $y_{0}$.

2.2. We next consider the parametrization of a path $x: I \rightarrow G$ by means of the arc length of its image $f \circ x$ under a mapping $f: G \rightarrow R^{n}$. The interval $I$ may be closed, half open or open. We shall use for paths the notation and terminology of [9, pp. 1-8]. Thus $l(x)$ is the length of a rectifiable closed path $\alpha:[a, b] \rightarrow R^{n}, s_{\alpha}:[a, b] \rightarrow[0, l(\alpha)]$ is the length function of $\alpha$, and $\alpha^{0}:[0, l(\alpha)] \rightarrow R^{n}$ is the normal representation of $\alpha$, satisfying $\alpha^{0} \circ s_{\alpha}=\alpha$. If $\alpha$ is the restriction of a path $\beta$ to a subinterval, we say that $\alpha$ is a subpath of $\beta$ and write $x \subset \beta$.

2.3. Lemma. Suppose that $f: G \rightarrow R^{n}$ is a light mapping. Suppose also that $\beta:[a, b] \rightarrow R^{n}$ is a rectifiable closed path and that $x: I \rightarrow G$ is a path such that $f \circ \alpha \subset \beta$. Then there is a unique path $1^{*}: s_{\beta} I \rightarrow G$ such that $\alpha=\alpha^{*} \circ\left(s_{\beta} \mid I\right)$. Moreover, $f \circ \alpha^{*} \subset \beta^{0}$.

Proof. The function $s_{\beta}:[a, b] \rightarrow[0, l(\beta)]$ is continuous and increasing. If $s_{\beta}\left(t_{1}\right)=s_{\beta}\left(t_{2}\right)$ for $t_{1}, t_{2} \in I$, then $\beta$ is constant on $\left[t_{1}, t_{2}\right]$. Since $f \circ \alpha \subset \beta$ and since $f$ is light, also $\alpha$ is constant on $\left[t_{1}, t_{2}\right]$. Hence there is a unique mapping $\alpha^{*}$ of $I^{*}=s_{\beta} I$ into $G$ such that $\alpha=\alpha^{*} \circ\left(s_{\beta} \mid I\right)$. The continuity of $\alpha^{*}$ follows easily from the continuity of $\alpha$. If $t \in I$, then $f\left(\alpha^{*}\left(s_{\beta}(t)\right)\right)=f(\alpha(t))=\beta(t)=\beta^{0}\left(s_{\beta}(t)\right)$. Hence $f \circ \lambda^{*}=\beta^{0} I^{*}$.

2.4. Definition. Suppose that $f: G \rightarrow R^{n}$ is a light mapping and that $\alpha: I \rightarrow G$ is a closed path. We say that $f$ is absolutely precontinuous on $\alpha$ if $\beta=f \circ \alpha$ is rectifiable and if the path $\alpha^{*}:[0, l(\beta)] \rightarrow G$, given by 2.3 , is absolutely continuous. If the path $\mathrm{i}$ is open or half open, we say that $f$ is absolutely precontinuous on $\alpha$ if it is absolutely precontinuous on every closed subpath of $\alpha$.

2.5. Remarks. (1) If $f$ is a homeomorphism onto a domain $G^{\prime}$, then $f$ is absolutely precontinuous on $\alpha$ if and only if $f^{-1}$ is (locally) absolutely continuous on $f \circ \alpha$. 
(2) We shall make use of the following elementary observation: In the situation of Lemma 2.3, the path $\alpha^{*}$ depends on the path $\beta$, but only up to a translation of the parameter. More precisely, if $f \circ \alpha \subset \beta_{1}$ and $f \circ x \subset \beta_{2}$, and if $\alpha_{1}^{*}, \alpha_{2}^{*}$ are the corresponding paths given by 2.3, then $\alpha_{1}^{*}(t)=\alpha_{2}^{*}\left(t+t_{0}\right)$ for some constant $t_{0}$. In particular, $\alpha^{*}$ is (locally) absolutely continuous if and only if $f$ is absolutely precontinuous on $x$.

2.6. Lemma. Suppose that $f: G \rightarrow R^{n}$ is quasiregular. Let $\Gamma_{0}$ be the family of all paths $\beta$ in $R^{n}$ such that either $\beta$ is non-rectifiable or there is a path $\alpha$ in $G$ such that $f \circ x \subset \beta$ and $f$ is not absolutely precontinuous on a. Then $M\left(\Gamma_{0}\right)=0$.

Proof. This lemma is a slight extension of an important result of Poleckii [7, Lemma 6]. The topological part of Poleckii's proof has been simplified by Rickman [8]. Choose an exhaustion $\left(G_{i}\right)$ of $G$. This means that $G_{1}, G_{2}, \ldots$ is a sequence of domains such that $\bar{G}_{i} \subset G_{i+1}$ and $G=$ $\cup\left\{G_{i}: i \in N\right\}$. Let $\Gamma_{i}$ be the family of all closed paths in $G_{i}$ on which $f$ is not absolutely precontinuous. By the aforementioned result of Poleckii, $M\left(f \Gamma_{i}\right)=0$. Furthermore, the family $\Gamma_{\text {non }}$ of all non-rectifiable paths is of modulus zero. Since $\Gamma_{0}$ is minorized by the union of $\Gamma_{\text {non }}$ and all $f \Gamma_{i}, M\left(\Gamma_{0}\right)=0$.

\section{Modulus and capacity inequalities}

3.1. Theorem. Suppose that $f: G \rightarrow R^{n}$ is a non-constant quasiregular mapping, that $I^{\prime}$ is a path family in $G$, that $\Gamma^{\prime}$ is a path family in $R^{n}$, and that $m$ is a positive integer such that the following condition is satisfied:

There is a set $E_{0} \subset G$ of measure zero such that for every path $\beta: I \rightarrow R^{n}$ in $\Gamma^{\prime}$ there are paths $x_{1}, \ldots, x_{m}$ in $\Gamma$ such that $f \circ x_{i} \subset \beta$ for all $i$ and such that for every $x \in G \backslash E_{0}$ and $t \in I, \backslash_{i}(t)=x$ for at most one $i$.

Then

$$
M\left(\Gamma^{\prime}\right) \leq \frac{K_{I}(f)}{m} M(\Gamma)
$$

3.3. Remarks. It is not required that $\Gamma^{\prime}=f \Gamma$. We shall later apply the theorem with $E_{0}=B_{f}$. If $\Gamma^{\prime}=f \Gamma$, the condition is trivially true for $m=1$, and we obtain Poleckii's inequality $M(f T) \leq K_{I}(f) M(T)$. If $D$ is a normal domain of $f$, if $\Gamma^{\prime}$ is a family of paths in $f D$, and if $\Gamma$ is the family of all paths $x$ in $D$ such that $f \circ x \in \Gamma^{\prime}$, then the condition is satisfied for $m=N(f, D)$ by Rickman [8]. Hence we obtain Poleckii's second inequality (1.2). 
3.4. Proof of Theorem 3.1. Let $\Gamma_{0}$ be the family of Lemma 2.6. Setting $\Gamma_{1}=\Gamma^{\prime} \backslash \Gamma_{0}$ we have $M\left(\Gamma_{1}\right)=M\left(\Gamma^{\prime}\right)$. Hence it suffices to prove that

$$
M\left(\Gamma_{1}\right) \leq \frac{K_{I}(f)}{m} M(\Gamma) .
$$

We may assume that $E_{0}$ is a Borel set. By [4, 8.2], we may also assume that at all points $x \in G \backslash E_{0}, f$ is differentiable and $J(x, f)>0$. Thus $B_{f} \subset E_{0}$. Let $\varrho \in F(T)$. Define $\sigma: G \rightarrow \dot{R}^{1}$ by

$$
\begin{aligned}
& \sigma(x)=\varrho(x) / l\left(f^{\prime}(x)\right) \quad \text { for } \quad x \in G \backslash E_{0} . \\
& \sigma(x)=\infty \quad \text { for } x \in E_{0} \text {. }
\end{aligned}
$$

Then $\sigma$ is a Borel function. Next define $\varrho^{\prime}: R^{n} \rightarrow \dot{R}^{1}$ by

$$
\varrho^{\prime}(y)=m^{-1} \sup _{B} \sum_{x \in B} \sigma(x),
$$

where $B$ runs through all subsets of $f^{-1}(y)$ such that card $B \leq m$. For $y \in \mathbf{C} f G$ put $\varrho^{\prime}(y)=0$. Then $\varrho^{\prime}(y)=\infty$ for $y \in f E_{0}$.

We shall prove that $\varrho^{\prime} \in F\left(\Gamma_{1}\right)$. To show that $\varrho^{\prime}$ is a Borel function, we choose an exhaustion of $G$ with domains $G_{i}, \bar{G}_{i} \subset G_{i+1}$. Denoting by $\chi_{A}$ the characteristic function of a set $A$, we set

$$
\varrho_{i}=\varrho \chi \bar{G}_{i}, \sigma_{i}=\sigma \chi_{\bar{G}_{i}}, \varrho_{i}^{\prime}(y)=m^{-1} \sup _{B} \sum_{x \in B} \sigma_{i}(x)
$$

As $i \rightarrow \infty, \varrho_{i}(x) \rightarrow \varrho(x)$ and $\varrho_{i}^{\prime}(y) \rightarrow \varrho^{\prime}(y)$ for all $x \in G$ and $y \in R^{n}$. Furthermore, $\varrho_{i}^{\prime}(y)=0$ for $y \in \mathbf{C} f \bar{G}_{i}$, and $\varrho_{i}^{\prime}(y)=\infty$ for $y \in f\left(\bar{G}_{i} \cap B_{f}\right)$. Hence it suffices to show that every $y_{0} \in f \bar{G}_{i} \backslash f\left(\bar{G}_{i} \cap B_{f}\right)$ has a neighborhood in which $f$ is a Borel function. Apply Lemma 2.1 with $E=\bar{G}_{i}$, and let $V$ be the corresponding neighborhood of $y_{0}$. Setting $g_{j}=f_{j}^{-1}$, we have

$$
\varrho_{i}^{\prime}(y)=m^{-1} \sup _{J} \sum_{j \in J} \sigma_{i}\left(g_{j}(y)\right)
$$

for $y \in V$, where $J$ runs through all subsets of $\{1, \ldots, k\}$ such that card $J \leq m$. Since every $\sigma_{i}^{\circ} g_{j}$ is a Borel function, $\varrho_{i}^{\prime} V$ is a Borel function.

Next let $\beta$ be a member of $\Gamma_{1}$. We must show that

$$
\int_{\beta} \varrho^{\prime} d s \geq 1 \text {. }
$$

Assume first that $\beta:[a, b] \rightarrow R^{n}$ is a closed path. By the hypothesis, there are paths $\alpha_{1}, \ldots, \alpha_{m}$ in $\Gamma$ such that $f \circ \alpha_{i} \subset \beta$ and such that card $\left\{i \mid \alpha_{i}(t)=x\right\} \leq 1$ for all $x \in G \backslash E_{0}$ and $t \in[a, b]$. Set $c=l(\beta)$, 
$\gamma=\beta^{0}$, and let $\gamma_{i}: I_{i} \rightarrow G$ be the path $\alpha^{*}$ given by Lemma 2.3 for $\alpha=\alpha_{i}$. Thus $\alpha_{i}(t)=\gamma_{i}\left(s_{\beta}(t)\right)$ and $f \circ \gamma_{i} \subset \gamma$.

For almost every $t \in[0, c]$ we have $\left|\gamma^{\prime}(t)\right|=1$ by [9, 1.3.(5)]. Since $f$ is absolutely precontinuous on each $\alpha_{i}$, the paths $\gamma_{i}$ are (locally) absolutely continuous (Remark 2.5.(2)). Hence the derivative $\gamma_{i}^{\prime}(t)$ exists a.e. in $I_{i}$. It follows that for almost every $t \in I_{i}$, either $\gamma_{i}(t) \in E_{0}$ or

$$
1=\left|\gamma^{\prime}(t)\right|=\left|f^{\prime}\left(\gamma_{i}(t)\right) \gamma_{i}^{\prime}(t)\right| \geq l\left(f^{\prime}\left(\gamma_{i}(t)\right)\right)\left|\gamma_{i}^{\prime}(t)\right| .
$$

Since $\sigma(x)=\infty$ for $x \in E_{0}$, the inequality $\sigma\left(\gamma_{i}(t)\right) \geq \varrho\left(\gamma_{i}(t)\right)\left|\gamma_{i}^{\prime}(t)\right|$ holds a.e. in $I_{i}$. Consequently (cf. $[9,4.1]$ ),

$$
1 \leq \int_{\alpha_{i}} \varrho d s=\int_{\gamma_{i}} \varrho d s=\int_{I_{i}} \varrho\left(\gamma_{i}(t)\right)\left|\gamma_{i}^{\prime}(t)\right| d t \leq \int_{I_{i}} \sigma\left(\gamma_{i}(t)\right) d t,
$$

$1 \leq i \leq m$. Set $h_{i}(t)=\sigma\left(\gamma_{i}(t)\right) \chi_{I_{i}}(t)$ for $t \in[0, c]$, and let $J(t)=$ $\left\{i \mid t \in I_{i}\right\}$. For every $t \in[0, c]$, either $\gamma(t) \in f E_{0}$, in which case $\varrho^{\prime}(\gamma(t))=\infty$, or the points $\gamma_{i}(t), i \in J(t)$, are distinct points in $f^{-1}(\gamma(t))$. In both cases we have

$$
\varrho^{\prime}(\gamma(t)) \geq m^{-1} \sum_{i=1}^{m} h_{i}(t),
$$

which implies

$$
\int_{\beta} \varrho^{\prime} d s=\int_{0}^{c} \varrho^{\prime}(\gamma(t)) d t \geq m^{-1} \sum_{i=1}^{m} \int_{0}^{c} h_{i}(t) d t=m^{-1} \sum_{i=1}^{m} \int_{I_{i}} \sigma\left(\gamma_{i}(t)\right) d t \geq 1 .
$$

If the path $\beta$ is open or half open, we can apply the above proof to the closed extension $[9,3.2]$ of $\beta$. We have proved that $\varrho^{\prime} \in F\left(\Gamma_{1}\right)$. Consequently,

$$
M\left(\Gamma_{1}\right) \leq \int \varrho^{\prime n} d m .
$$

To estimate the above integral, we again choose an exhaustion $\left(G_{i}\right)$ of $G$ and introduce the functions $\varrho_{i}, \sigma_{i}$ and $\varrho_{i}^{\prime}$ as in (3.5). Fix $i$, let $y_{0} \in f \bar{G}_{i} \backslash f\left(\bar{G}_{i} \cap B_{f}\right)$, and let $V$ be a connected neighborhood of $y_{0}$ satisfying the conditions of Lemma 2.1 for $E=\bar{G}_{i}$. We have thus $k$ homeomorphisms $g_{j}: V \rightarrow D_{j}, \quad f \circ g_{j}=\mathrm{id}, \quad$ and $\quad \bar{G}_{i} \cap f^{-1} V=\bigcup\left\{\bar{G}_{i} \cap D_{j} \mid\right.$ $1 \leq j \leq k\}$. Put $J_{0}=\{1, \ldots, k\}$, and define for each $y \in V$ a set $J(y) \subset J_{0}$ as follows: If $k \leq m$, then $J(y)=J_{0}$. If $k>m$, then card $J(y)=m$, and for all $j \in J(y), j^{\prime} \in J_{0} \backslash J(y)$, either $\sigma_{i}\left(g_{j}(y)\right)>$ $\sigma_{i}\left(g_{j^{\prime}}(y)\right)$ or $\sigma_{i}\left(g_{j}(y)\right)=\sigma_{i}\left(g_{j^{\prime}}(y)\right)$ and $j>j^{\prime}$. Then

$$
\varrho_{i}^{\prime}(y)=m^{-1} \sum_{j \in J(y)} \sigma_{i}\left(g_{j}(y)\right)
$$


for $y \in V$. For $J \subset J_{0}$, the sets $V_{J}=\{y \in V \mid J(y)=J\}$ are disjoint Borel sets. Using Hölder's inequelity, a transformation formula for Lebesgue integrals, and the quasiconformality of $f \mid D_{j}$, we obtain

$$
\begin{aligned}
\int_{V_{J}} \varrho_{i}^{\prime n} d m & \leq m^{-1} \sum_{j \in J} \int_{V_{J}} \sigma_{i}\left(g_{j}(y)\right)^{n} d m(y) \\
& =m^{-1} \sum_{j \in J_{g_{j} V_{J}}} \frac{\varrho_{i}(x)^{n}}{l\left(f^{\prime}(x)\right)^{n}} J(x, f) d m(x) \\
& \leq \frac{K_{I}(f)}{m} \int_{f^{-1} V_{J}} \varrho_{i}^{n} d m .
\end{aligned}
$$

Summing over all $J \subset J_{0}$ yields

$$
\int_{V} \varrho_{i}^{\prime n} d m \leq \frac{K_{I}(f)}{m} \int_{f^{-1 V}} \varrho_{i}^{n} d m .
$$

Since $\varrho_{i}^{\prime}(y)=0$ for $y \in \mathbf{C} f \bar{G}_{i}$ and since $f \bar{G}_{i} \backslash f\left(\bar{G}_{i} \cap B_{f}\right)$ can be almost covered by a countable number of disjoint sets $V$ as above (for example, with cubes), and since $m\left(f B_{f}\right)=0$, we obtain

$$
\int \varrho_{i}^{\prime n} d m \leq \frac{K_{I}(f)}{m} \int \varrho_{i}^{n} d m
$$

As $i \rightarrow \infty$, this and (3.7) yield

$$
M\left(T_{1}\right) \leq \frac{K_{I}(f)}{m} \int Q^{n} d m
$$

Since $\varrho \in F(\Gamma)$ was arbitrary, this proves the theorem.

3.8. Examples. Let us consider the complex analytic function $f: R^{2} \rightarrow$ $R^{2}, f(z)=e^{z}$. Let $m$ be a positive integer, and let $Q$ be the rectangle $0<\operatorname{Re} z<1,0 \leq \operatorname{Im} z<2 \pi m$. Then $f Q$ is the annulus $B^{2}(e) \backslash \bar{B}^{2}$. Let $\Gamma$ be the family of all horizontal segments of line joining the vertical sides of $Q$. It is easy to see that the condition of Theorem 3.1 is satisfied for $\Gamma$ and $\Gamma^{\prime}=f \Gamma$. Hence $M\left(\Gamma^{\prime}\right) \leq M(\Gamma) / m$. On the other hand, it is well known that $M\left(\Gamma^{\prime}\right)=2 \pi=M(\Gamma) / m$. Hence the inequality (3.2) is sharp in this case. This also follows from the inequality $[4,3.2] M(I) \leq$ $N(f, Q) K_{o}(f) M(f \Gamma)$.

Next let $\Gamma$ be the family of all vertical segments joining the horizontal sides of $Q$. Now we have $M(f I)=1 / 2 \pi m^{2}=M(\Gamma) / m$. Hence (3.2) 
is also true in this case. However, the condition of Theorem 3.1 is not satisfied. We shall give a result which applies to situations like this.

If $\alpha:[a, b] \rightarrow G$ is a closed path, we say that $f$ winds $\alpha m$ times around itself if $f \circ \alpha=\beta$ is rectifiable and if the following condition is satisfied: Let $\beta^{0}:[0, c] \rightarrow R^{n}$ be the normal representation of $\beta$, let $\alpha^{*}:[0, c] \rightarrow G$ be the path given by 2.3 , and let $h=c / m$. Then $\beta^{0}(t+j h)=\beta^{0}(t) \quad$ and $\quad \alpha^{*}(t+j h) \neq \alpha^{*}(t) \quad$ whenever $\quad 0 \leq t<t+j h<c$ and $j \in\{1, \ldots, m-1\}$.

3.9. Theorem. Suppose that $f: G \rightarrow R^{n}$ is a non-constant quasiregular mapping, that $\Gamma$ is a path family in $G$, that $m$ is a positive integer, and that $f$ winds every path of $\Gamma m$ times around itself. Then

$$
M(f \Gamma) \leq \frac{K_{I}(f)}{m} M(T) .
$$

Proof. The proof is closely similar to the proof of Theorem 3.1. The only difference is the proof of the inequality

$$
\int_{\beta} \varrho^{\prime} a s \geq 1
$$

for $\beta=f \circ x \in f \Gamma$. Now

$$
\int_{\beta} \varrho^{\prime} d s=m \int_{0}^{h} \varrho^{\prime}\left(\beta^{0}(t)\right) d t
$$

If $0<t<h$, then $\alpha^{*}(t), \alpha^{*}(t+h), \ldots, x^{*}(t+(m-1) h)$ are distinct points in $f^{-1}\left(\beta^{0}(t)\right)$. Hence

$$
\underline{Q}^{\prime}\left(\beta^{0}(t)\right) \geq m^{-1} \sum_{j=0}^{m-1} \sigma\left(\lambda^{*}(t+j h)\right)
$$

for $t \in(0, h)$. As in the proof of 3.1 we obtain $\sigma\left(\chi^{*}(t)\right) \geq g\left(\chi^{*}(t)\right) \mid \alpha^{* \prime}(t)$ for almost every $t \in[0, c]$. Consequently,

$$
\int_{\beta} \varrho^{\prime} d s \geq \sum_{j=0}^{m-1} \int_{0}^{h} \varrho\left(\alpha^{*}(t+j h)\right)\left|\chi^{* \prime}(t)\right| d t=\int_{\alpha} \varrho d s \geq 1 .
$$

This proves (3.6).

3.10. Remark. The situation of 3.9 arises in the theory of covering mappings. Suppose that $f$ is a quasiregular covering mapping of $G$ onto $G^{\prime}$ such that the fundamental group $\pi_{1}\left(G^{\prime}\right)$ is isomorphic to the group $Z$ 
of integers. Suppose that $\Delta$ is a path family in $G^{\prime}$ such that every member of $\Delta$ is a rectifiable loop which represents a generator of $\pi_{1}\left(G^{\prime}\right)$. Let $m$ be a positive integer such that $m \leq N(f)$. For each $\gamma \in \Delta, \gamma:[a, b] \rightarrow G^{\prime}$, choose a point $x \in f^{-1}(\gamma(a))$, and let $\alpha$ be the path in $G$ obtained by performing $m$ successive liftings of $\gamma$, the first one starting at $x$. Then $f$ winds $\propto m$ times around itself. To see this, we may assume that $\gamma:[0, h] \rightarrow G^{\prime}$ is a normal representation. Then $\alpha:[0, m h] \rightarrow G$ is a path with the property $f(\alpha(t+j h))=\gamma(t)$ for $0 \leq t \leq h$ and $1 \leq j \leq m-1$. If $\alpha(t+j h)=\alpha(t)$ for some $t$ and $j \leq m-1$, the path $\alpha_{1}=\alpha \mid[t, t+j h]$ is a loop such that $f \circ \alpha_{1}$ represents an element $q$ in $\pi_{1}\left(G^{\prime}\right)=Z$ such that $|q|=j$. However, this is impossible, because the induced homomorphism $f_{*}$ maps $\pi_{1}(G)$ onto $N(f) Z$ if $N(f)<\infty$, and onto $\{0\}$ if $N(f)=\infty[2,15.4$, p. 88].

Let $\Gamma$ be the family of all liftings $\alpha$. By 3.9 , we have

$$
M(f T) \leq \frac{K_{I}(f)}{m} M(\Gamma) .
$$

This inequality can be written in another form. In fact, a function $\varrho$ belongs to $F(f \Gamma)$ if and only if $m \varrho \in F(\Delta)$. Hence $M(\Delta)=m^{n} M(f \Gamma)$, which yields

$$
M(\Delta) \leq m^{n-1} K_{I}(f) M(\Gamma) .
$$

3.11. Maximal liftings. We shall need some results concerning path lifting for discrete open mappings. If $f: G \rightarrow R^{n}$ is a mapping, if $\beta$ : $[a, b) \rightarrow R^{n}$ is a path and if $x_{0} \in f^{-1}(\beta(a))$, we say that a path $\alpha:[a, c) \rightarrow G$ is a maximalf-lifting of $\beta$ starting at $x_{0}$ if $\alpha(a)=x_{0}, f \circ x \subset \beta$ and there does not exist a path $\alpha_{1}:\left[a, c_{1}\right) \rightarrow G$ such that $x \subset x_{1}$ and $f \circ \alpha_{1} \subset \beta$. See [6, p. 12]. The following result is from Rickman [8]:

3.12. Lemma. Suppose that $f: G \rightarrow R^{n}$ is discrete and open, that $\beta$ : $[a, b) \rightarrow R^{n}$ is a path and that $x_{1}, \ldots, x_{k}$ are points in $f^{-1}(\beta(a))$. Set $m=\sum_{j=1}^{k} i\left(x_{j}, f\right)$. Then there are maximal f-liftings $\alpha_{1}, \ldots, \alpha_{m}$ of $\beta$ such that

(1) $\operatorname{card}\left\{j \mid x_{j}(a)=x_{i}\right\}=i\left(x_{i}, f\right)$ for $\quad 1 \leq i \leq k$.

(2) card $\left\{j \mid \alpha_{j}(t)=x\right\} \leq i(x, f)$ for all $x \in G$ and $t \in[a, b)$.

3.13. Condensers. A condenser in $R^{n}$ is a pair $E=(A, C)$ where $A \subset R^{n}$ is open and $C \subset A$ is compact. See [4, p. 24]. The capacity of a condenser $E=(A, C)$ is the number

$$
\operatorname{cap} E=\inf _{u} \int_{A} \mid \nabla u^{n} d m
$$


where $u$ runs through all $C^{\infty}$-functions with compact support in $A$ such that $u(x) \geq 1$ for $x \in C$. An alternative way to define the capacity of $E$ is the equality

$$
\operatorname{cap} E=M\left(\Gamma_{E}\right) \text {, }
$$

where $\Gamma_{E}$ is the family of all paths joining $C$ and $\partial A$ in $A$. This was proved by Ziemer $[10,3.8]$ for bounded condensers, and the general case can be established by a simple limiting process. For our purposes, it is most convenient to let $\Gamma_{E}$ be the family of all balf open paths $\gamma:[a, b) \rightarrow A$ such that $\gamma(a) \in C$ and $\gamma(t) \rightarrow \partial A$ as $t \rightarrow b$, cf. [9, 11.3]. If $f: G \rightarrow R^{n}$ is an open mapping and if $E=(A, C)$ is a condenser in $G$, then $f E=(f A, f C)$ is also a condenser. In $[4,7.1]$ it was proved that

$$
\operatorname{cap} f E \leq K_{I}(f) \operatorname{cap} E
$$

for non-constant quasiregular mappings $f$. Martio $[3,5.1]$ proved the inequality

$$
\operatorname{cap} f E \leq \frac{K_{I}(f) N(f, A)^{n-1}}{M(f, C)^{n}} \operatorname{cap} E .
$$

Here $M(f, C)$ is the minimal multiplicity of $f$ on $C$, defined by

$$
M(f, C)=\inf _{y \in f C} \sum_{x \in f^{-1}(y) \cap C} i(x, f) .
$$

Since $1 \leq M(f, C) \leq N(f, A)$ by $[3,3.6]$, the inequalities (3.15) and (3.16) are consequences of the following result:

3.17. Theorem. Suppose that $f: G \rightarrow R^{n}$ is a non-constant quasiregular mapping and that $E=(A, C)$ is a condenser in $G$. Then

$$
\operatorname{cap} f E \leq \frac{K_{I}(f)}{M(f, C)} \operatorname{cap} E .
$$

Proof. Set $\Gamma=\Gamma_{E}, \Gamma^{\prime}=\Gamma_{f E}$, and $m=M(f, C)$. Let $\beta:[a, b) \rightarrow f A$ be a path in $\Gamma^{\prime}$. Then $C \cap f^{-1}(\beta(a))$ contains points $x_{1}, \ldots, x_{k}$ such that $\sum\left\{i\left(x_{j}, f\right) \mid 1 \leq j \leq k\right\} \geq m$. By 3.12, there are maximal $(f \mid A)$-liftings $\alpha_{j}:\left[a, c_{j}\right) \rightarrow G$ of $\beta, 1 \leq j \leq m$, such that $\alpha_{j}(a)=x_{i}$ for some $i$ and such that card $\left\{j \mid \alpha_{j}(t)=x\right\} \leq 1$ for $x \in G \backslash B_{f}$ and $t \in[a, b)$. Furthermore, it follows from [6, 3.12] that $\alpha_{j}(t) \rightarrow \partial A$ as $t \rightarrow c_{j}$. Hence $\alpha_{j} \in \Gamma$ for all $j$. The theorem follows from 3.1 and (3.14).

3.18. Remark. We sketch a proof of 3.17 , which does not make use of path families. Let $S$ be the set of all pairs $(x, k)$ such that $x \in A$ and $1 \leq k \leq i(x, f), k \in N$. Let $P: S \rightarrow A$ be the projection $P(x, k)=x$. 
Using the notation of [4, p. 25], we define for every $u \in \mathrm{I}_{0}^{\infty}(E)$ a function $v: f A \rightarrow R^{1}$ by

$$
v(y)=M(f, C)^{-1} \max _{B} \sum_{z \in B} u(P(z)),
$$

where $B$ runs through all subsets of $P^{-1}\left(f^{-1}(y)\right)$ such that card $B \leq$ $M(f, C)$. Modifying the proofs of $[4,7.1]$ and $[3,5.1]$, we can show that $v \in W_{0}(f E)$ and that

$$
\int|\nabla v|^{n} d m \leq \frac{K_{I}(f)}{M(f, C)} \int \mid \nabla u^{n} d m .
$$

3.19. Remark. All the results of this section can be extended without difficulties to quasimeromorphic mappings $f: G \rightarrow \bar{R}^{n}[5,2.1]$.

\section{Applications}

4.1. Let $f: G \rightarrow R^{n}$ be a quasiregular mapping. An isolated boundary point $b$ is said to be an isolated singularity of $f$. Furthermore, $b$ is a removable singularity, a pole, or an essential singularity according as $f$ has a finite limit, an infinite limit, or no limit at $b$. See [5, p. 12].

4.2. Theorem. Suppose that $b$ is an isolated singularity of a quasiregular mapping $f: G \rightarrow R^{n}$. Suppose also that there are finite positive constants $C, p, \delta$ such that

$$
|f(x)| \leq C x^{-p}
$$

for $0<|x-b|<\delta$. Then $b$ is not an essential singularity of $f$.

Proof. Assume that $b$ is an essential singularity. Performing a preliminary similarity transformation, we may assume that $b=0$, that $\bar{B}^{n} \subset G \cup\{0\}$, and that $C=\delta=1$. Choose $R>0$ such that $f S^{n-1} \subset$ $B^{n}(R)$. From [5, 4.6] it follows that there is $y_{0}$ in $R^{n}$ such that $\left|y_{0}\right|>R$ and $N\left(y_{0}, f, B^{n}\right)=\infty$. Set $K=K_{I}(f)$, choose a positive integer $m$ such that $m>2 K p^{n-1}$, and choose distinct points $x_{1}, \ldots, x_{m}$ in $B^{n} \cap f^{-1}\left(y_{0}\right)$. Applying [4, 2.9], we next choose $r>0$ such that $U_{i}=$ $U\left(x_{i}, f, r\right)$ is a normal neighborhood of $x_{i}$ for $1 \leq i \leq m$, the closures $\bar{C}_{i}$ are disjoint, and $\bar{U}_{i} \subset B^{n}$. Set $d=d\left(0, \bar{C}_{1} \cup \ldots \cup \bar{C}_{m}\right)$, and let $a \in(0, d)$. Let $V$ be the ring $B^{n} \backslash \bar{B}^{n}(a)$. Since $f$ is open, $\partial f V \subset f \partial V=$ $=f S^{n-1} \cup f S^{n-1}(a) \subset B^{n}(R) \cup \bar{B}^{n}\left(a^{-p}\right)$. Since $y_{0} \in f V$, this implies $f V \subset$ $B^{n}\left(a^{-p}\right)$.

Consider the hemisphere $H=\left\{e \in S^{n-1} \quad\left(e: y_{0}\right)>0\right\}$. Let $\Gamma^{\prime}$ be the family of all paths $\beta:\left[r, a^{-p}\right) \rightarrow R^{n}$, defined by $\beta(t)=y_{0}+t e$, 
$e \in H$, and let $\Gamma$ be the family of all maximal $(f \mid V)$-liftings of the members of $T^{\prime}$, starting at points of $\bar{U}_{1} \cup \ldots \cup \bar{U}_{m}$. Then 3.1 and $3.12 \mathrm{imply}$

$$
M\left(\Gamma^{\prime}\right) \leq \frac{K}{m} M\left(I^{\prime}\right) .
$$

Suppose that $\alpha:[r, c) \rightarrow G$ is a member of $\Gamma$. Since $g V \subset B^{n}\left(a^{-p}\right)$. $y_{0}+a^{-p} e \in \mathbf{C} f V$ for all $e \in H$. From [6, 3.12] it follows that $\alpha(t) \rightarrow \partial V$ as $t \rightarrow c$. This means that either $|\alpha(t)| \rightarrow 1$ or $|\alpha(t)| \rightarrow a$. The first case is impossible, because $\overline{|\beta|} \cap f S^{n-1}=\varnothing$ for all $\beta \in \Gamma^{\prime}$. Hence $|\alpha(t)| \rightarrow a$ as $t \rightarrow c$. Hence $\Gamma$ is minorized by the family $\Gamma_{1}$ of all paths joining the spheres $S^{n-1}(d)$ and $S^{n-1}(a)$. Consequently,

$$
M(I) \leq M\left(\Gamma_{1}\right)=\omega_{n-1}\left(\log \frac{d}{a}\right)^{1-n} .
$$

On the other hand, by $[9,7.7]$ we have

$$
M\left(\Gamma^{\prime}\right)=\frac{1}{2}()_{n-1}\left(\log \frac{a^{-p}}{r}\right)^{--n} \text {. }
$$

Combining the above inequalities yields

$$
a^{q} \geq r d^{(m / 2 K)^{1 /(n-1)}},
$$

where

$$
q=\left(\frac{m}{2 K}\right)^{1 /(n-1)}-p>0 .
$$

As $a \rightarrow 0$, this gives a contradiction.

4.3. Theorem. Suppose that $b$ is an isolated singularity of a quasiregular mapping $f: G \rightarrow R^{n}$, and let $\alpha=K_{I}(f)^{1 /(1-n)}$. If $\lim _{x \rightarrow b}|x-b|^{\alpha}|f(x)|$ $=0, b$ is a removable singularity. The hypothesis cannot be replaced by the requirement that $|x-b|^{\alpha}|f(x)|$ be bounded in a neighborhood of $b$.

Proof. We may assume that $b=0$. By $4.2, b$ cannot be an essential singularity. Assume that $b$ is a pole of $f$. Let $g$ be a Möbius transformation of $\bar{R}^{n}$ such that $|g(x)|=1 /|x|$ for all $x \in \bar{R}^{n}$. Then $h=g \circ f$ is quasiregular in a neighborhood of 0 , and $K_{I}(h)=K_{I}(f)$. From $[5,3.2]$ it follows that $|h(x)| \leq C|x|^{\alpha}$ in a neighborhood of 0 , where $C$ is a constant. Hence $|x|^{\alpha}|f(x)| \geq 1 / C$, which contradicts the hypothesis. Thus 0 is a removable singularity of $f$.

The mapping $f(x)=g\left(|x|^{\alpha-1} x\right)$, where $g$ is as above, has a pole at the origin, $K_{I}(f)=\alpha^{1-n}$, and $|x|^{\alpha}|f(x)|=1$ for all $x \in R^{n} \backslash\{0\}$. 
4.4. Remark. In the special case $n=2, K(f)=1$, the theorems 4.2 and 4.3 are well known results for analytic functions [1, p. 124 and p. 128].

\section{References}

1. Ahlfors, L. V.: Complex analysis, 2nd ed. - McGraw-Hill, 1966.

2. Hu, S.-T.: Homotopy theory. - Academic Press, 1959.

3. Martio, O.: A capacity inequality for quasiregular mappings. - Ann. Acad. Sci. Fenn. A I 474 (1970), 1-18.

4. Martio, O., Rickman, S., and J. VärsäLÄ: Definitions for quasiregular mappings. - Ann. Acad. Sci. Fenn. A I 448 (1969), 1-40.

5. - - Distortion and singularities of quasiregular mappings. - Ann. Acad. Sci. Fenn. A I 465 (1970), 1-13.

6. - - Topological and metric properties of quasiregular mappings. - Ann. Acad. Sci. Fenn. A I 488 (1971), 1-31.

7. Polecki, Е. А. (Полецкий, Е. А.): Метод модулей для негомеоморфных квавиконформных отображений. - Mat. Sbornik 83 (1970), $261-272$.

8. Rickman, S.: Path lifting for discrete open mappings. - To appear.

9. VäIsäL $\ddot{A}$, J.: Lectures on $n$-dimensional quasiconformal mappings. - SpringerVerlag, 1971.

10. Ziemer, W. P.: Extremal length and p-capacity. - Michigan Math. J. 16 (1969), $43-51$.

University of Helsinki

Helsinki, Finland 